\title{
Oligonucleotide-Hybridization Fiber-Optic Biosensor using a Narrow Bandwidth Long Period Grating
}

\author{
Martina Delgado-Pinar, Qing Shi, Luis Poveda-Wong, Estefanía Delgado-Pinar, Baojian Xu, Jianlog \\ Zhao, Jose Luis Cruz, and Miguel V. Andrés
}

\begin{abstract}
Nanometric narrowband Long Period Gratings (LPG) are investigated for the implementation of improved fiber optic biosensors. The reduction of more than one order of magnitude in the linewidth of the LPG with respect standard LPG at $1500 \mathrm{~nm}$, leads to the improvement of the resolution of the sensor. By selecting the proper fabrication parameters (high numerical aperture, relatively high order mode and large length), LPGs with a 3-dB bandwidth of $1.5 \mathrm{~nm}$ were fabricated. The sensitivity of the LPG as a refractometer was calculated, and experimentally characterized in detail. In particular, the $\mathbf{L P}_{0,17}$ and $L_{0,18}$ resonances were investigated, in order to select the most suitable one for the bioexperiments. The surface of the LPG was functionalized and the detection of the hybridization of DNA is demonstrated. When the biosensor was immersed in a $2 \mu \mathrm{M}$ solution of the complementary DNA strand, the resonances of the LPG shifted in wavelength. When measuring the response of the sensor in terms of variation of its transmittance as the resonance shifted in wavelength, the sensitivity was $\sim 10 \% / \mu \mathrm{M}$ for both resonances. The detection limit was estimated in $10 \mathrm{nM}$.
\end{abstract}

Index Terms-Fiber optics, Fiber optics sensors, Fiber Gratings, Biosensors.

\section{INTRODUCTION}

$\mathrm{T}$ HE development of photonic biosensors has been a rapid growth research field in the last years. In particular, the development of fiber-optic, label-free biosensors is of special interest: they can be designed to be specific and selective, they show compact size and are capable to be point-of-care devices [1-3]. Within the applications of fiber-optic biosensors, the detection of DNA hybridization is of much interest, due to their specific capability to detect particular DNA sequences that might be of interest for environmental, biological or

This work is funded by projects iPhotoBio (PIRSES-GA-2013-612267, EU) and UV-INV-AE16-485280 (Universitat de València).

M. Delgado-Pinar, L. Poveda-Wong, J. L. Cruz and M. V. Andrés are with the Departamento de Física Aplicada y Electromagnetismo - ICMUV, Universidad de Valencia, 46100, Valencia, Spain (e-mail: martina.delgado@uv.es, $\quad$ luis.poveda@uv.es, miguel.andres@uv.es ).

Q. Shi, B. Xu and J.Zhao are with the Shanghai Institute of Microsystem and Information Technology (SIMIT), Chinese Academy of SciencesShanghai Tech. University, China (e-mail : shiqing@shanghaitech.edu.cn, xbj@mail.sim.ac.cn,jlzhao@mail.sim.ac.cn)

E. Delgado-Pinar is with the ICMOL, Universitat de València, C/Catedrático José Beltrán 2, 46980 Paterna, Spain (e-mail: estefania.delgado@uv.es). health applications, epidemic controls, diagnosis, drug research, etc. Several approaches have been reported up to date to measure the DNA-hybridization based on different photonic devices, as for example micro-fiber gratings [4], gold-coated microtapers [5], surface plasmon resonances based sensors [6], microwave photonic filters [7] or dual-peak Long Period Gratings (LPGs) [8]. Other sensors based on waveguides with strong evanescent fields show also high potential as, for example, reverse symmetry waveguides $[9,10]$.

LPGs are fiber devices whose optical properties rely on the resonant coupling between the fundamental mode and the proper cladding modes. The coupling is induced by a periodic modulation inscribed in the core refractive index, whose parameters set the optical response of the LPG. It will present a series of attenuation notches, corresponding to the coupling to different cladding modes. The bandwidth of the attenuation notches can be tailored within some limitations. Typical bandwidths are in the range from several to tens of nanometers. In a previous work, we demonstrate that LPGs with a reduced bandwidth down to the order of $1 \mathrm{~nm}$ can be fabricated [11]. In this way, the detection limit of a biosensor based on these narrowband LPGs is improved, since the difficulty of determining with accuracy the wavelength of resonance in a broad attenuation notch is overcome.

LPGs show an intrinsic advantage as sensors, compared to other similar fiber photonic devices as FBGs: the cladding modes coupled by the LPG have a significant evanescent tail in the surrounding medium, thus the sensitivity to external changes is higher. Any modification in the external refractive index will modify the optical response of the LPG, in particular, the spectral position of the resonances. In general, the sensitivity will change for LPGs fabricated with different parameters, and it will also be different for each resonance of the same LPG, depending on the dispersion curve of the cladding modes involved in the coupling. Refractometers, solution concentration sensors and E. Coli biosensors have been demonstrated based on this sensing principle [12-15].

In this work, we propose the use of a narrowband LPG to measure the DNA hybridization in an aqueous solution. LPGs are simple, robust photonic devices when compared with the other approaches we mentioned before, which are based on a few microns in diameter fibers [4-7]. The unusual narrow bandwidth of the LPGs we present here allows improving the detection limit of this biosensor when compared to dual-peak 
LPGs [8], while keeping a good sensitivity thanks to the dispersion properties of the modes involved in the coupling. The fabrication parameters of the LPGs were chosen in order to get a good sensitivity of the resonances as a function of the external refractive index, and good coupling coefficients, while keeping its bandwidth in the order of $1 \mathrm{~nm}$. Two LPGs with two resonances in the $1400-1600 \mathrm{~nm}$ spectral range (corresponding to the cladding modes $\mathrm{LP}_{0,17}$ and $\mathrm{LP}_{0,18}$ ) were fabricated and tested. First, the sensing performance of the resonances was measured in detail as a function of the external refractive index. The second grating was tested as an oligonucleotide hybridization biosensor. The response of the biosensor can be read in terms of wavelength shift of the resonance, or in terms of the variation of the transmittance at a given wavelength within the bandwidth of the resonance. The sensitity, resolution and detection limit of each approach will be measured and analyzed

\section{MATERIALs, Design AND FABRICATION OF THE BIOSENSOR}

\section{A. Materials}

(3-Aminopropyl)triethoxysilane (APTES), $1-$ methylimidazole $99 \%$, hydrochloric acid $(\mathrm{HCl})$, and SSPE buffer were purchased from Fisher Scientific SL (Spain). N(3-Dimethylaminopropyl)-N'-ethylcarbodiimide hydrochloride (EDC) was purchased from Sigma-Aldrich (Spain). Water was twice distilled and passed through a Millipore apparatus.

The oligonucleotides (Eurogentec, Spain) probes were designed to include a phosphate group at the 5'-ends. The sequence of basis was chosen the same as the one reported in [8], in order to compare the performance of our biosensor with previous results, as follows,

Probe: 5'-phosphate-GCA CAG TCA GTC GCC-3'

Complementary: 5'-GGC GAC TGA CTG TGC-3'

\section{B. Design and fabrication of the narrowband $L P G$}

A periodic refractive index modulation inscribed in the core of a section of fiber, allows the resonant coupling between the fundamental mode and those cladding modes with the proper symmetry. The coupling will happen when the phase matching condition for the two modes is fulfilled:

$$
\lambda_{R}=\Lambda \cdot\left(n_{c o}-n_{c l}\right)
$$

where $\lambda_{R}$ is the resonant wavelength of the coupling, $\Lambda$ is the period of the modulation, $n_{c o}$ is the effective index of the fundamental mode and $n_{c l}$ is the effective index of the cladding mode involved in the coupling.

The evanescent tail of the cladding modes is in contact with the external medium that will contain the analyte; its presence, or the variation of its concentration, will lead to a change in $n_{c l}$. As a consequence, a wavelength shift of the resonance will be observed, see Fig. 1. The sensitivity of each resonance will depend on the dispersion curves of the modes involved in the coupling. The wavelength shift of the spectral notch of an LPG when a perturbation $\Delta n_{\text {ext }}$ is introduced in the external medium will be given by

$$
\Delta \lambda_{\mathrm{R}}=\Lambda \cdot \frac{\frac{\partial \mathrm{n}_{\mathrm{cl}}}{\partial \mathrm{n}_{\mathrm{ext}}}}{1-\frac{\Lambda}{\lambda_{\mathrm{R}}} \cdot \Delta \mathrm{n}_{\mathrm{g}}} \cdot \Delta \mathrm{n}_{\mathrm{ext}}
$$

where $n_{\text {ext }}$ is the refractive index of the surrounding medium, and $\Delta n_{g}$ stands for the group index difference between the coupled modes.

In addition, the 20-dB-bandwidth of each attenuation peak will be given by:

$$
\Delta \lambda_{3 \mathrm{~dB}} \approx \frac{0.8 \cdot \lambda_{\mathrm{R}}}{\mathrm{L} \cdot \Delta \mathrm{n}_{\mathrm{g}}} \approx \frac{0.8 \cdot \Lambda}{\mathrm{N}}
$$

where $L$ is the length of the LPG, and $N$ is the number of periods [11]. Then, within some limits, it is possible to design a LPG with a narrow bandwidth and a good sensitivity by choosing properly its parameters. In general, in order to obtain a narrow bandwidth, fibers with high NA should be employed: the higher index core results in gratings of smaller period, this is, a higher number of periods $N$, which reduces the bandwidth. The order of the cladding mode determines the grating bandwidth as well. However, as our purpose is to use the LPG as a biosensor, we also have to take into account (a) its sensitivity and (b) the possibility of achieving deep resonances. This last condition requires coupling the light to relatively high order cladding modes. Roughly, modes $\mathrm{LP}_{0,15}$ to $\mathrm{LP}_{0,20}$ present a good coupling coefficient with the fundamental mode of the fiber [11]; however the bandwidth of the resonance $\mathrm{LP}_{0,20}$ would be too wide for our purpose, since it is already close to the turning point of the dispersion curve. It should be noted, as well, that the bandwidth scales with the resonant wavelength. As a consequence, it is more challenging fabricating a narrow bandwidth LPG with the resonance within the $1500 \mathrm{~nm}$ spectral region than at shorter wavelengths.

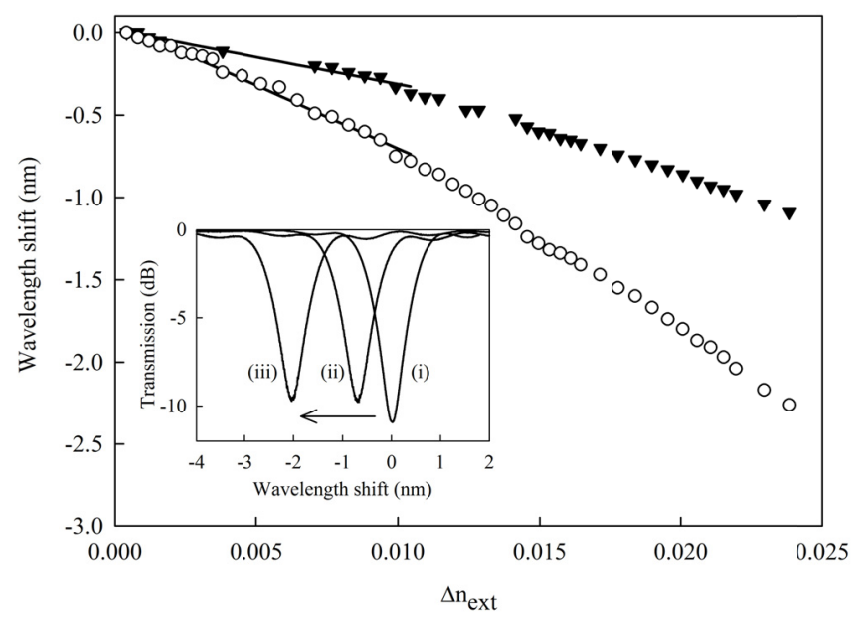

Fig. 1. Wavelength shift of the resonances as a function of $\Delta n_{\text {ext }}$. Triangles: $\mathrm{LP}_{0,17}$; circles: $\mathrm{LP}_{0,18}$ mode. Inset: experimental spectra of the $\mathrm{LP}_{0,18}$ resonance for (i) $\Delta \mathrm{n}_{\mathrm{ext}}=0$, (ii) $\Delta \mathrm{n}_{\mathrm{ext}}=1.04 \times 10^{-2}$, (iii) $\Delta \mathrm{n}_{\mathrm{ext}}=2.2 \times 10^{-2}$.

The LPGs used for the experiments were photoinscribed in a high numerical aperture $(\mathrm{NA}=0.29)$, highly germanium doped fiber (SM1500 Fibercore), by using a CW frequency doubled Ar laser (244 nm), and the point-by-point technique. 
The fiber had a cutoff wavelength of $1387 \mathrm{~nm}$, a modal field diameter of $4.2 \mu \mathrm{m}$ and an attenuation of $1.95 \mathrm{~dB} / \mathrm{Km}$ at 1550 $\mathrm{nm}$. The period of the grating was set to $52 \mu \mathrm{m}$, and the writing process was stopped when the length of the LPG was $\sim 10 \mathrm{~cm}$. The birefringence of the SM1500 fiber causes that the LPG response shows polarization dependence, thus polarizers and polarization controllers had to be included in the setup.

Firstly, we studied the sensing performance of a narrowband LPG as a refractometer, see Fig. 1. A first narrowband grating, $12 \mathrm{~cm}$ in length, was used for this experiment. The notch correspondent to $\mathrm{LP}_{0,18}$ resonance was centered at $1523.0 \mathrm{~nm}$ and showed a $3-\mathrm{dB}$ bandwidth $\left(\Delta \lambda_{3 \mathrm{~dB}}\right)$ of $1.05 \mathrm{~nm}$; its minimum transmission was $-12.6 \mathrm{~dB}$. The following notch, correspondent to the $\mathrm{LP}_{0,17}$ resonance, appeared at $1400.0 \mathrm{~nm} \quad\left(\Delta \lambda_{3 \mathrm{~dB}}=0.66 \mathrm{~nm}, \quad\right.$ minimum transmission $-11.3 \mathrm{~dB}$ ). No other couplings were observed in this range, thus the free spectral range of the LPG was larger than $120 \mathrm{~nm}$. The polarization of the optical signal was adjusted to the slow axis of the fiber for all the measurements. All the spectra of the LPG in this paper were measured using an optical spectrum analyzer (OSA) with $20 \mathrm{pm}$ resolution.

In order to monitor the blue wavelength shift of the resonances as the external refractive index is increased, this LPG was immersed in an ethanol-water mixture, whose refractive index was modified by adding ethanol. To estimate the refractive index increment of the mixture, we used the formula reported in [16]. Figure 1 shows the experimental results: the circles correspond to the measurement of the blue shift of the $\mathrm{LP}_{0,18}$ resonance as the ethanol is added, while the triangles are for the $\mathrm{LP}_{0,17}$ resonance. In order to illustrate how the biosensor operates, the inset in Fig. (1) shows a series of experimental spectra of the $\mathrm{LP}_{0,18}$ resonance for different external refractive index variations: (i) $0,0 \mathrm{RIU}$ increment (original LPG in water), (ii) $1.04 \times 10^{-2} \mathrm{RIU}$ increment, (iii) $\Delta \mathrm{n}_{\mathrm{ext}}=2.2 \times 10^{-2}$ RIU increment.

It should be note that, with this technique, there is a large refractive index range along which the central wavelength of the resonance can be monitored without ambiguity, due to the large free spectral range between resonances (larger than $120 \mathrm{~nm}$ ). This feature is an intrinsic advantage of this technique when compared to others which are based on interferometers, in which the transmittance of the refractometer shows a periodic pattern of fringes, thus a careful measurement is required in order to avoid the ambiguity produced by the different periods of the output signal.

The data show a clearly different sensitivity between the two resonances. For low index variations (less than 0.01 ), both curves show a linear dependence, see the linear fits included in the figure. The slope of these linear fits correspond to the sensitivity of each resonance: $-32.8 \pm 1.5 \mathrm{~nm} / \mathrm{RIU}$ for the $\mathrm{LP}_{0,17}$ resonance, and $-77 \pm 2 \mathrm{~nm} / \mathrm{RIU}$ for $\mathrm{LP}_{0,18}$. We calculated the sensitivity of the resonances for the range of low refractive index variation (this is, less than 0.01 RIU, where the resonances shift linearly). For the simulations, we used the refractive index of the water at the corresponding wavelength as the unperturbed external refractive index, which was calculated taking into account the chromatic dispersion. For the $\mathrm{LP}_{0,18}$ resonance, the calculated sensitivity is $-94 \mathrm{~nm} / \mathrm{RIU}$ at $1530 \mathrm{~nm}$, while it is $-43.8 \mathrm{~nm} / \mathrm{RIU}$ for the $\mathrm{LP}_{0,17}$ resonance around $1400 \mathrm{~nm}$. The values of the experimental sensitivities agree reasonably with our theoretical calculations. The difference between the calculated and experimental sensitivities may have its origin in a number of reasons (for example, the formula reported in [16] for the estimation of the refractive index of the ethanol/water mixture is measured at $633 \mathrm{~nm}$, while we are measuring in the infrared range). It should be noted that there are other modes that show better sensitivity, especially when considering dual-peak LPGs (values of $794 \mathrm{~nm} / \mathrm{RIU}$ have been reported [8]). However, the bandwidth of the resonances corresponding to these modes is in the order of tens of nm, which limits the detection limit of the sensor. Then, to improve the general performance of the biosensor, a compromise between sensitivity and resolution must be achieved. For our experiments with DNA, we chose the fabrication parameters to have LPGs of $\sim 1 \mathrm{~nm}$ bandwidth around $1.55 \mu \mathrm{m}$, although this comes with the drawback of the relative long lengths $(\sim 10 \mathrm{~cm})$, since the length and the bandwidth of the resonances are inversely proportional. In order to reduce the length while keeping the bandwidth $\sim 1 \mathrm{~nm}$, the biosensor could be designed to operate at shorter wavelengths, see Eq. (3).

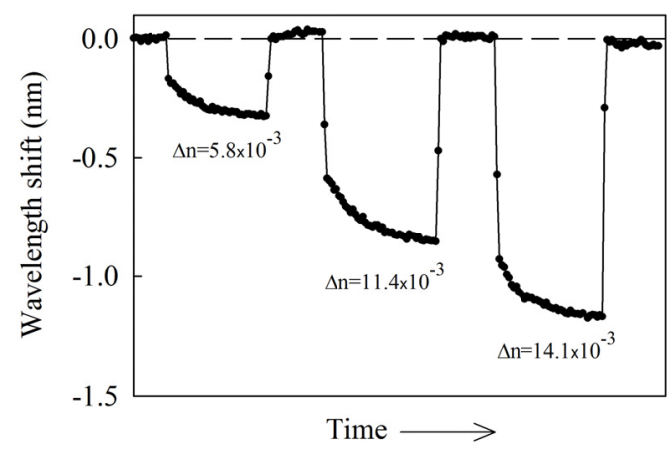

Fig. 2. Temporal evolution of the wavelength shift for different $\Delta n_{\text {ext }}$.

The central wavelength of the $\mathrm{LP}_{0,18}$ resonance was measured as a function of time, for different concentrations of ethanol, this is, different $\Delta \mathrm{n}_{\text {ext }}$. The results are shown in Fig. 2. The resonance shifted to a shorter wavelength when the ethanol-water mixture was pumped into the cuvette containing the LPG, and the signal was stable after a few minutes (the noise in the wavelength position at this point was less than $10 \mathrm{pm}$ ). When pure water replaced the ethanol-water mixture, the resonance returned to its original position. The process was repeated three times, for three different concentrations, and the wavelength shifts agreed with the sensitivity measured in Fig. 1.

A second narrowband LPG was fabricated to perform the DNA hybridization sensing experiments. When the polarization was adjusted to the slow axis of the fiber, the 3-dB bandwidth $\left(\Delta \lambda_{3 \mathrm{~dB}}\right)$ of the notch centered in $1548.9 \mathrm{~nm}$ (corresponding to $\mathrm{LP}_{0,18}$ mode) was $1.52 \mathrm{~nm}$, and the minimum transmission was $-9.2 \mathrm{~dB}$. A second notch centered at $1415.8 \mathrm{~nm}\left(\mathrm{LP}_{0,17}\right.$ mode) was observed. In this case, the minimum transmission was $-11.3 \mathrm{~dB}$ and the $\Delta \lambda_{3 \mathrm{~dB}}$ was 0.82 
$\mathrm{nm}$. The free spectral range of this LPG was larger than $130 \mathrm{~nm}$. Figure 3 shows the measured spectra of the fabricated LPG (black lines) for both resonances, and the calculated spectra (dashed lines), when the external medium is air. The parameters employed for the simulation were: $\Lambda=52 \mu \mathrm{m}$, $\mathrm{L}=70 \mathrm{~mm}$ (the effective length is shorter than the experimental value), and the modulation depth of the core refractive index was set to $\sim 1.7 \times 10^{-5}$. It can be observed that the experimental notch for the $\mathrm{LP}_{0,18}$ mode is broader than expected. This could be due to the overlapping of the central notch with undesired sidelobes that grew systematically and invaded the main resonance broadening the spectrum. This was probably due to imperfections in the fabrication process; several factors must been taken into account: temperature variation during the fabrication process (typically $\sim 1^{\circ} \mathrm{C}$ ), power variations of the UV laser $( \pm 2 \mathrm{~mW})$ or irregularities of the fiber that could change the difference between effective indices up to $10^{-6}$. We estimated that the overall effect in the bandwidth of the resonances is an rms error of $\pm 110 \mathrm{pm}$ along the grating.
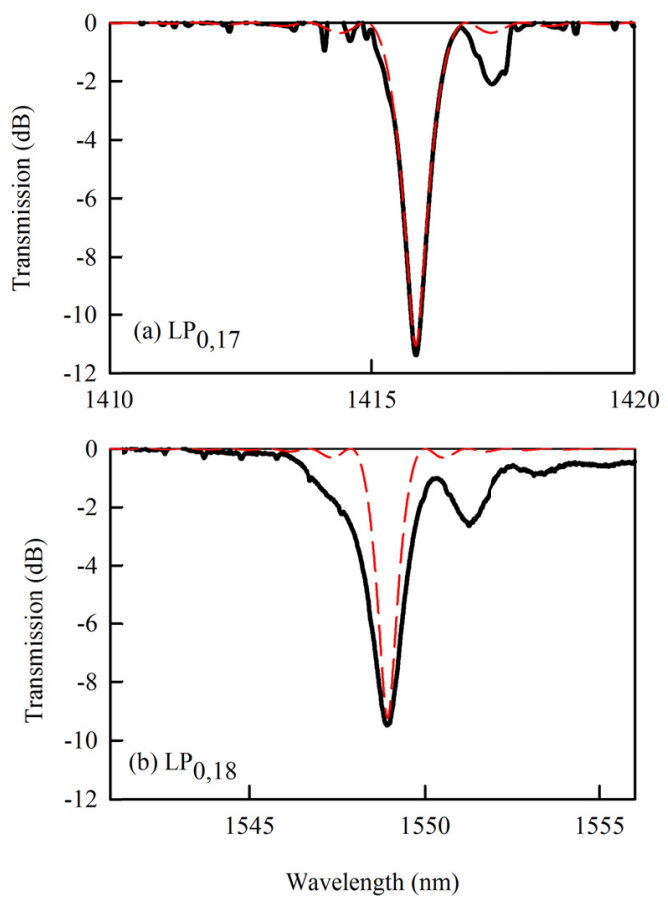

Fig. 3. Transmission spectra of the LPG, experimental (continuous line) and simulation (dashed line), for modes (a) $\mathrm{LP}_{0,17}$ and (b) $\mathrm{LP}_{0,18}$.

Additionally to the LPG, a short FBG $(\sim 10 \mathrm{~mm})$ was inscribed scanning a UV beam over the fiber through a phase mask, at a distance of $\sim 15 \mathrm{~mm}$ from one end of the LPG (thus, the total length of the biosensor was shorter than $150 \mathrm{~mm}$ ). This FBG was centered at $1561.7 \mathrm{~nm}$, far from the attenuation notches of the LPG, and its bandwidth was $200 \mathrm{pm}$. This FBG was included in the device as a reference, in order to monitor any thermal or strain changes that could affect the measurements of the LPG's wavelength shift. If the biosensor were used in a thermally controlled environment, this additional FBG would not be necessary, resulting in a shorter length of the biosensor.
As we said, the detection limit of the sensor is improved as the bandwidth of the resonance decreases. In our case, from the experiments of the sensing performance, we could conclude that the resolution of our measurement equipment was a wavelength shift of $10 \mathrm{pm}$, which is, roughly, a $1 \%$ part of the resonance bandwidth (this value is consistent with the noise observed in the experiment shown in Fig. 2). This resolution corresponds, according to our experimental sensitivities, to a detection limit of $\Delta n_{\text {ext }} \sim 1.3 \times 10^{-4}$ for the $\mathrm{LP}_{0,18}$ resonance (see Ref. [17] for the definitions of resolution and detection limit of the biosensor). This value is comparable to that obtained by Falate et al. [13], where they present a refractometer based on a phase-shifted LPG inscribed in a pure-silica-core fiber $\left(\sim 2.1 \times 10^{-3}\right)$. However, their sensitivity $(-20 \mathrm{~nm} / \mathrm{RIU})$ is lower than ours.

The response of the sensor can also be encoded in terms of amplitude variations of a laser signal tuned to the central point of the edge of the LPG, where the transmission slope is at its maximum. In our case, the steep slope of the narrowband resonance leads to a better detection limit when comparing with broader LPGs. A further advantage is that the measurement of power does not require specific equipment to detect wavelength variations. In addition, a feedback system could be introduced in the setup, in order to keep the laser tuned at the central point of the edge of the resonance, as it shifts in wavelength. With this improvement, we could take full advantage of the optical and electronic possibilities of our proposal, and the range of external refractive indices that can be measured with this technique is as large as in the previous case (when measuring wavelength shifts).

In order to estimate the detection limit for this approach, we will assume that the resolution in terms of power is a thousandth part of the normalized transmittance at the point of maximum transmission slope (this is, $0.1 \%$ ); in this case, our $\mathrm{LP}_{0,18}$ resonance provides a detection limit of $1.7 \times 10^{-5} \mathrm{RIU}$ $\left(3.0 \times 10^{-5}\right.$ RIU for the $\mathrm{LP}_{0,17}$ one). These values are comparable to those obtained by Falate et al. $\left(6.8 \times 10^{-5}\right.$ RIU) [13], and those reported by Cao et al., $\left(2.6 \times 10^{-5}\right.$ RIU) [7], where they report a microwave photonic filter using microfibers to detect DNA hybridization. It is worth to note that the performance of our sensor is simpler than theirs, while providing similar or better detection limits.

\section{Surface functionalization of the biosensor.}

The method employed for the functionalization of the surface of the fiber is described in detail in [8]. The process consists of two stages: first, a silanization of the surface using APTES and, second, an EDC-mediated oligonucleotide immobilization, where the EDC acts as a catalyst. Shortly, the region of the grating fiber was immersed in a freshly prepared $0.1 \mathrm{M} \mathrm{HCl}$ solution for $30 \mathrm{~min}$, rinsed with DI water and air dried. The anchoring of the probe-DNA to the surface of the fiber was achieved by direct hydrolysis of the $-\mathrm{OH}$ groups located all around the surface of the fiber, and the hydrolysable alkyl groups bonded to the silicon atom present in the APTES reagent. To do so, the glass fiber was soaked in an ethanolic solution of APTES for 12 hours allowing the anchoring of the primary amine on the surface of the fiber, rinsed with DI water and air-dried. At the second stage, the immobilization of the DNA was carried out by condensation 
of the primary amine group with the organic phosphate present in the 5'-oligonucleotide sequence through a phosphoramidate linkage. A buffered aqueous solution $(10 \times$ PBS) containing the 5'-phosphorylated oligonucleotide (5'-phosphate-GCA CAG TCA GTC GCC-3') (5 $\mu \mathrm{M})$, 1-methylimidazole (30 $\mathrm{mM})$ and EDC hydrochloride $(65 \mathrm{mM})$ was added and kept under stirring for a couple of minutes. The amino-silanized glass fiber was soaked in this solution and after $24 \mathrm{hrs}$ incubation, the fiber was rinsed with buffer solution, DI water, and air-dried.
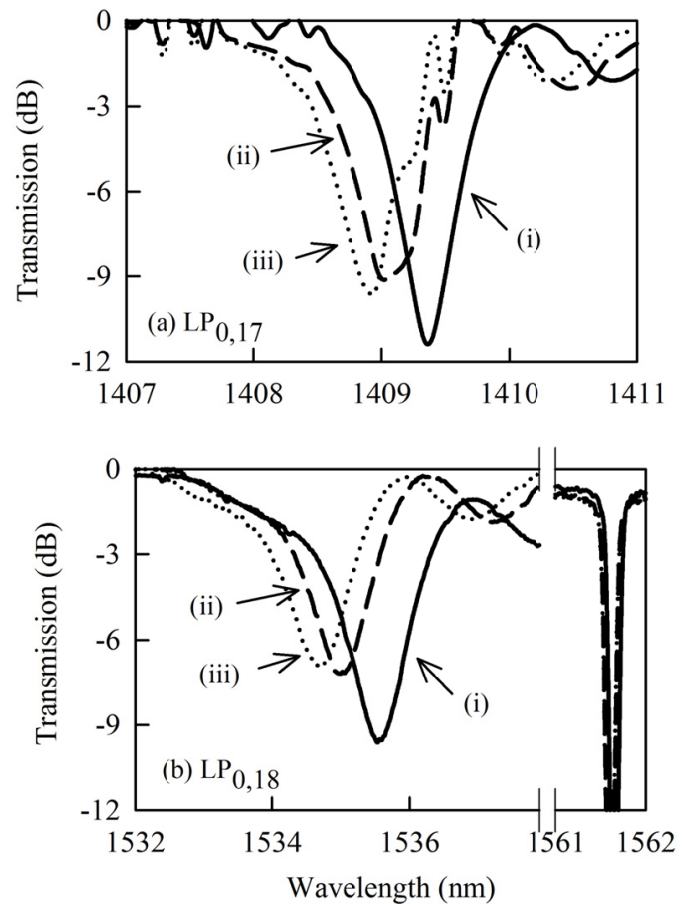

Fig. 4. Transmission spectra of the LPG for both resonances: (i) original LPG and FBG (continuous line), (ii) after silanization (dashed line), (iii) after immobilization of the DNA-probe (dotted line).

Figure 4 shows the spectra of both resonances of the LPG and the FBG, (i) after cleaning the fiber and immersed in the buffer, (ii) after silanization, and (iii) after oligonucleotideprobe immobilization. A polarized LED source from 1370 to $1600 \mathrm{~nm}$ was used to illuminate the LPG. The spectral position of the FBG was tested over time while the chemical reactions took place, and it remained constant during the process. The LPG spectra show that there is a significant shift in both resonances after each stage. These shifts indicate that the chemical processing of the fiber was successful, and it introduced a modification on the surface that could be resolved by the LPG acting as a sensor. The shifts were different for each resonance, accordingly to the different sensitivities. Although the functionalization modifies the shape of the resonances, mainly by decreasing the coupling strength, the bandwidth is still in the order of $1 \mathrm{~nm}(1.20 \mathrm{~nm}$ for the $\mathrm{LP}_{0,18}$ resonance and $0.86 \mathrm{~nm}$ for the $\mathrm{LP}_{0,17}$ one), and the maximum attenuation exceeded $6 \mathrm{~dB}$ in the worst case.

Additionally, EDX analysis of the surface of different sections of functionalized fibers, and untreated sections, were done. The ratio of the carbon present on the surface between treated-untreated sections is 8:1, which confirms that the oligonucleotide was attached to the surface.

\section{HYBRIDIZATION OF THE COMPLEMENTARY OLIGONUCLEOTIDE}

After its functionalization, the biosensor was immersed in a freshly prepared 10× SSPE buffer solution with a final concentration of DNA-complementary strand of $2 \mu \mathrm{M}$. The transmission spectrum of the LPG was measured over time, and again the spectral position of the FBG was remained at a fixed wavelength all over the process. The maximum shift in wavelength of the LPG resonances is shown in Fig. 5: $-0.32 \mathrm{~nm}$ for the $\mathrm{LP}_{0,17}$ resonance and $-0.50 \mathrm{~nm}$ for the $\mathrm{LP}_{0,18}$ one. Therefore, the sensitivity of the biosensor in terms of concentration of the complementary oligonucleotide is $250 \mathrm{pm} / \mu \mathrm{M}$ for the $\mathrm{LP}_{0,18}$ resonance $(160 \mathrm{pm} / \mu \mathrm{M}$ for the $\mathrm{LP}_{0,17}$ ), value which is smaller than the one reported by Chen et al. in a factor of 2, although their reported sensitivity is about 8 times higher [8]. It should be noted that, when comparing this wavelength shift with those measured in Fig. 1, this DNA concentration would be the equivalent to an external refractive index variation of $6.5 \times 10^{-3}$ RIU. This first experiment about the sensing performance of the LPG showed that we could expect a linear behavior of our biosensor up to 0.01 RIU increment. Thus, we would expect that this biosensor could operate linearly for a DNA concentration range up to $\sim 3 \mu \mathrm{M}$.
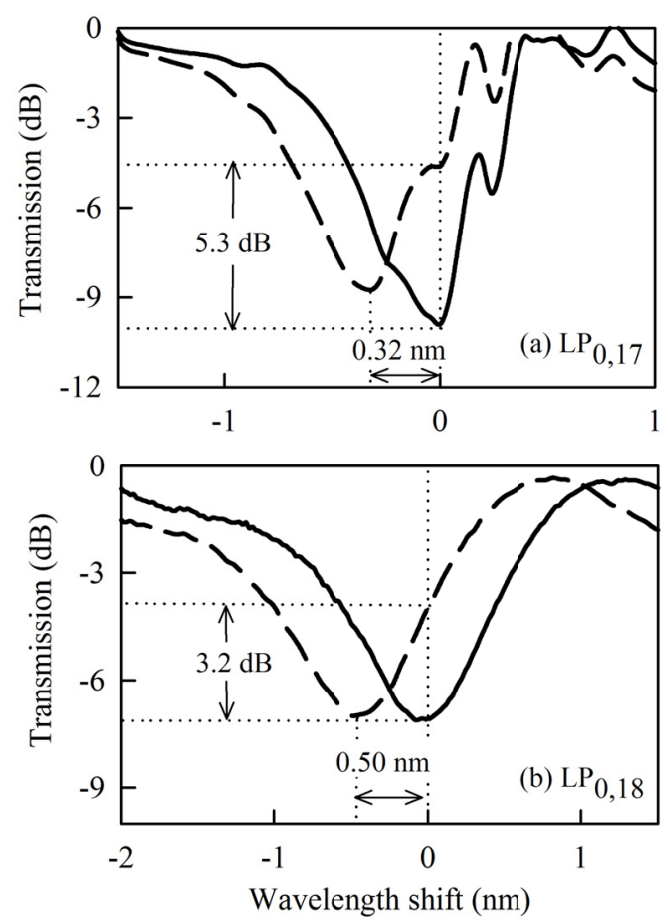

Fig. 5. Transmission spectra of the LPG for both resonances (a) $\mathrm{LP}_{0,17}$ and (b) $\mathrm{LP}_{0,18}$, along the hybridization process of the oligonucleotide: (i) initial spectra (continuous line), (ii) final spectra (dashed line).

Again, to estimate the detection limit of the biosensor, we considered that the resolution of the biosensor is $10 \mathrm{pm}$, which is the minimum wavelength shift we could observe with 
accuracy with our measurement equipment (this is, $1 \%$ of the bandwidth of the resonance, roughly). Taking into account this criterion, the detection limit is estimated to be $40 \mathrm{nM}$ for the $\mathrm{LP}_{0,18}$ resonance $\left(63 \mathrm{nM}\right.$ for the $\left.\mathrm{LP}_{0,17}\right)$. This value is better than the one reported by Sun et al., $0.5 \mu \mathrm{M}$ [4]. Here, the authors use a grating inscribed in microfibers of 3-10 $\mu \mathrm{m}$ in diameter, and claim a sensitivity of $310 \mathrm{~nm} / \mathrm{RIU}$. Their higher sensitivity is due to the larger evanescent fields in the sensing length, but such a low diameter of the microfibers introduces a drawback because of mechanical weakness and complexity in the setup when compared to other approaches that use conventional fibers. In order to compare our results with those reported by Chen et al. [8], we assume that their resolution is, as in our case, a $1 \%$ of the resonance bandwidth $\left(\Delta \lambda_{3-\mathrm{dB}}=35\right.$ $\mathrm{nm}$ in their case). This leads to a detection limit value for their biosensor of $0.68 \mu \mathrm{M}$. Thus their proposal provides a better sensitivity at the expense of a higher detection limit.

If the response of the biosensor is coded in variations of transmittance as previously explained, the $2 \mu \mathrm{M}$ oligonucleotide solution leads to a variation of $5.3 \mathrm{~dB}$ or $24 \%$ (linear scale) of the transmittance for the $\mathrm{LP}_{0,17}$ resonance and $3.2 \mathrm{~dB}$ or $22 \%$ (linear scale) for $\mathrm{LP}_{0,18}$, as it is shown in Fig. 5 (note the different minimum transmission value of the resonances). Thus, the sensitivity of the biosensor in terms of variation of power is $12 \%$ and $11 \%$ per $\mu \mathrm{M}$ of DNA, respectively. We can compare our results with Leung et al., where they propose to use a tapered fiber optic $(5-10 \mu \mathrm{m}$ in diameter) coated with gold [5]. The sensitivity they claim is $\sim 11 \% / \mu \mathrm{M}$ of DNA, which is roughly the same as ours.

Regarding the detection limit, we will assume again that our resolution is a thousandth part of the normalized transmittance at the point of maximum transmission slope. With this value in mind, the detection limit of our biosensor is $10 \mathrm{nM}$. When compared with [5], their value is much better ( $750 \mathrm{fM})$, because of the larger evanescent field in the external medium. Again, we have to point out that the low diameter of the sensing devices is a drawback when this setup is compared to those that use fibers of conventional diameters. Finally, Chen et al. [8] report a sensitivity of $2 \% / \mu \mathrm{M}$ of oligonucleotide and, consequently, the estimated detection limit is $50 \mathrm{nM}$, this is, five times higher than ours.

Table 1 summarizes the results about sensitivity and detection limit in terms of concentration of DNAcomplementary for our biosensor.

TABLE I

SENSITIVITY AND DETECTION LIMIT OF THE BIOSENSOR

\begin{tabular}{cccc}
\hline \hline & & Sensitivity & Detection Limit \\
\hline \multirow{2}{*}{$\mathrm{LP}_{0,17}$} & Wavelength shift & $160 \mathrm{pm} / \mu \mathrm{M}$ & $63 \mathrm{nM}$ \\
& Amplitude variation & $12 \% / \mu \mathrm{M}$ & $10 \mathrm{nM}$ \\
& Wavelength shift & $250 \mathrm{pm} / \mu \mathrm{M}$ & $40 \mathrm{nM}$ \\
$\mathrm{LP}_{0,18}$ & Amplitude variation & $11 \% / \mu \mathrm{M}$ & $10 \mathrm{nM}$ \\
& & \\
\hline \hline
\end{tabular}

In order to check the reproducibility of our results, we repeated the whole process with an additional narrowband LPG $\left(\Delta \lambda_{3 \mathrm{~dB}}=1.42 \mathrm{~nm}\right.$ centered at $1519.4 \mathrm{~nm}$ for the $\mathrm{LP}_{0,18}$ coupling). We measured a wavelength shift of $0.4 \mathrm{~nm}$ for a $2 \mu \mathrm{M}$ concentration of oligonucleotide-complementary. Both results are similar. It is also worth to note that there are techniques to dehybridate the oligonucleotides, by increasing the temperature above the melting point or by the method reported in [18], that would allow reusing this type of biosensor

\section{CONCLUSIONS}

LPGs with a narrow bandwidth of about $1 \mathrm{~nm}$ have demonstrated to be suitable for the implementation of DNA-hybridization biosensors. LPG's resonances shift in wavelength as the DNA-complementary strand hybridizes to the DNA-probe attached to the surface of the fiber, since the evanescent wave of the cladding modes is in direct contact with the analyte. The development of narrow bandwidth LPGs is a novel approach to pursuit an improved detection limit while preserving good sensitivity. In this paper we studied both experimental and theoretically the sensing performance of the resonances correspondent to cladding modes $\mathrm{LP}_{0,17}$ and $\mathrm{LP}_{0,18}$, when the LPG works as a refractometer. The sensitivities of the resonances as a function of the complementary DNA concentration are $12 \% / \mu \mathrm{M}\left(\mathrm{LP}_{0,17}\right)$ and $11 \% / \mu \mathrm{M}\left(\mathrm{LP}_{0,18}\right)$. Our results confirm the possibility of achieving good detection limits (10 $\mathrm{nM}$ for both resonances) comparable to previous results in the literature, while using a robust and simple fiber component. Further improvements can be foreseen by optimizing the choice of cladding mode and fiber parameters, and by improving the setup in order to keep the environment conditions stabilized.

\section{REFERENCES}

[1] Leung A., Shankar P. M., Mutharasan R., "A review of fiber-optic biosensors," Sensors and Actuators B, Vol. 125 no. 2, pp. 688-703, 2007.

[2] Wang X. D., Wolfbeis O. S., "Fiber-optic chemical sensors and biosensors (2008-2012)," Anal. Chem., Vol 85, no. 2, pp. 487-508, 2013.

[3] Wolfbeis O. S., "Fiber-optic chemical sensors and biosensors," Anal. Chem., Vol. 88, no. 1, pp. 203-227, 2016.

[4] Sun D., Guo T., Ran Y., Huang Y., Guan B-O., "In-situ DNA hybridization detection with a reflective microfiber grating biosensor," Biosensors and Bioelectron., Vol. 61, no. 15, pp. 541-546, 2014.

[5] Leung A., Shankar P. M., Mutharasan R., "Label-free detection of DNA hybridization using gold-coated tapered fiber optic biosensors (TFOBS) in a flow cell at $1310 \mathrm{~nm}$ and $1550 \mathrm{~nm}$," Sensors and actuators, Vol. 131, no. 2, pp. 640-645, 2008

[6] Delport F., Pollet J., Janssen K., Verbruggen B., Knez K., Spasic D., Lammertyn J., "Real-time monitoring of DNA hybridization and melting processes using a fiber optic sensor," Nanotechnol., Vol. 23, no. 6, pp. $065503,2012$.

[7] Cao Y., Guo T., Wang X., Sun D., Rang Y., Feng X., Guan B-O., "Resolution-improved in situ DNA hybridization detection based on microwave photonic interrogation," Opt. Express, Vol. 23, no. 21, pp. 245326, 2015.

[8] Chen X., Liu C., Hughes M. D., Nagel D. A., Hine A. V., Zhang L., "EDC-mediated oligonucleotide immobilization on a long period grating optical biosensor," Biosensors \& Bioelectron., Vol. 6, no. 2, pp. 1000173,2015

[9] Horvath R. Pedersen H. C., "Demonstration of reverse symmetry waveguide sensing in aqueous solutions", App. Phys. Lett., Vol. 81, no. 12 , pp. 2166,2002

[10] Skivesen N., Horvath R., Pedersen H. C., "Multimode reverse-symmetry waveguide sensor for broad-range refractometry", Opt. Lett., Vol. 28, no. 24, pp. 2473-2475, 2003.

[11] L. Poveda-Wong, J. L. Cruz, M. Delgado-Pinar, X. Roselló-Mechó, A. Díez, M. V. Andrés, "Fabrication of long period gratings of subnanometric bandwidth," Opt. Lett., Vol. 2, no. 7, pp. 1265-1268, 2017. 
[12] Patrick H. J., Kersey A. D., Bucholtz F., "Analysis of the response of long period fiber gratings to external index of refraction," J. of Lightwave Technol., Vol. 16, no. 9, pp. 1606-1612, 1998.

[13] Falate R., Frazao O., Rego G., Fabris J.L., Santos J.L., "Refractometric sensor based on a phase-shifted long-period fiber grating," Appl. Opt., Vol. 45, no. 21, pp. 5066-5072, 2006.

[14] Falciai R., Mignani AG., Vannini A., "Long period gratings as solution concentration sensors," Sensors and Actuators B, Vol. 74, no. 1-3, pp. 74-77, 2001.

[15] K. Dandapat, S. M. Tripathi, Y. Chinifooroshan, W. J. Bock, and P. Mikulic, "Compact and cost-effective temperature-insensitive bio-sensor based on long-period fiber gratings for accurate detection of E. coli bacteria in water," Opt. Lett., Vol. 41, pp. 4198-4201, 2016.

[16] A. A. Ghoreyshi, F. A. Farhadpour, M. Soltanieh, and A. Bansal, "Transport of small polar molecules across nonporous polymeric membranes. I. Experimental procedures and data analysis", J. Membr. Sci., Vol. 211, pp. 193-214, 2003

[17] White I. M., Fan X., "On the performance quantification of resonant refractive index sensors," Opt. Express, Vol. 16, no. 2, pp. 1020-1028, 2008.

[18] Chen X., Zhang L., Zhou K., Davies E., Sugden K., Bennion I., Hughes M., Hine A., "Real-time detection of DNA interactions with long-period fiber-grating-based biosensor,", Opt. Lett., Vol. 32, no. 17, pp. 25412543, 2007. 


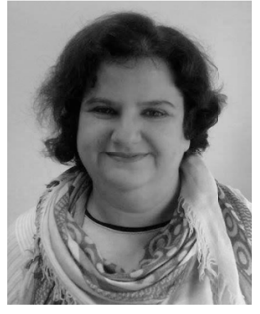

Martina Delgado-Pinar received the Licenciada en Física and the Ph.D. degrees from the Universitat de València (Spain) in 2002 and 2008, respectively. In 2008-2012 she worked as a postdoctoral research assistant at the Centre for Photonics and Photonic Materials of the University of Bath (UK). In 2012 she joined back to the Optical Fiber Laboratory of the Universitat de València as a Juan de la Cierva researcher. Currently, she is Assistant Teacher of the Departamento de Física Aplicada at this university. Her research topics include whispering gallery modes sensors, biosensors, tapered fibers, acousto-optic interaction, all-fiber lasers, nonlinear optics and fabrication of photonic crystal fibers.

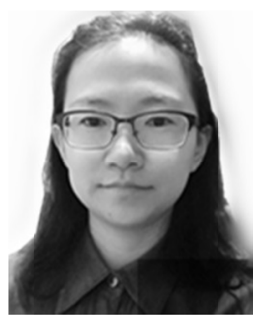

Qing Shi obtained the B.S. degree in biomedical engineering from Changchun University of Science and Technology in 2014, and was admitted in the State Key Laboratory of Transducer Technology of Shanghai Institute of Microsystem and Information Technology, Chinese Academy of Sciences. She is currently developing the research on photonic crystal waveguide for biosensing. Her interests include design and fabrication of optofluidic biochips, surface modification reactions and labelfree biosensors.

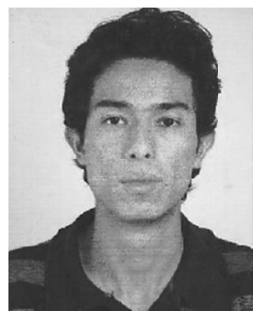

Luis Poveda-Wong was born in San José, Costa Rica, in 1987. He received the Electrical Engineering degree from the Universidad de Costa Rica in 2011. In 2013 he joined the Fiber Optics Group of the University of Valencia, where he is currently developing his dissertation on long period gratings for all-optical mathematical operators. His interests include fractional calculus applications, fiber Bragg grating sensors and ultrafast signal processing.

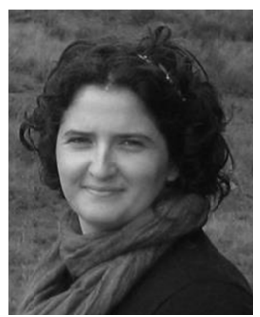

Estefanía Delgado-Pinar graduated in Chemistry at the University of Valencia in 2005. She achieved her PhD in Inorganic Chemistry in 2015. Her research focuses on modification of nanoparticles and other solid surfaces with organic molecules in order to achieve molecular recognition in water of charged species, using both surface science and supramolecular chemistry.

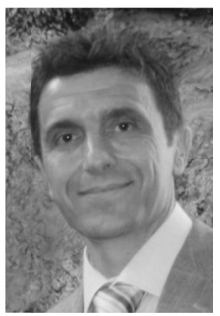

Jose Luis Cruz was born in Cuenca (Spain) in 1964. He received the $\mathrm{Ph}$. Degree in Physics from the University of Valencia (Spain) in 1992. Initially his career focused on microwave devices for radar applications, afterwards, he joined the Optoelectronics Research Center of the University of Southampton (UK) where he was working in optical fiber fabrication and he is currently Professor of the
Applied Physics Department at the University of Valencia where he is conducting research on fiber lasers and amplifiers fiber gratings, microwave photonics and sensors. He is coinventor of six patents related with the above mentioned topics, has published over 140 papers in international journals and more than 200 in conferences. He has also been ViceDean of the Physics Faculty and was Director of the Applied Physics Department for six years.

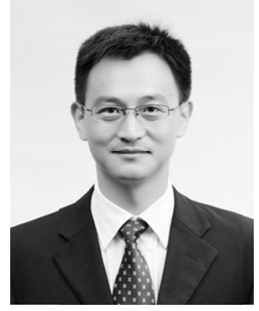

Baojian Xu received the Bachelor and $\mathrm{Ph}$. D degrees from Nanjing University and Shanghai Institute of Microsystem and Information Technology (SIMIT), in 2001 and 2006. At first, he served as an Assistant Professor in State Key Laboratory of Transducer Technology of SIMIT. Then, he worked as a visiting scholar in Hong Kong Polytech University from 2007 to 2009. In 2010, he joined back to State Key Laboratory of Transducer Technology of SIMIT as an Associate Professor. His research interests include Biomedical Microelectromechanical systems (BioMEMS), nanotechnology for biosensing and microfluidics.

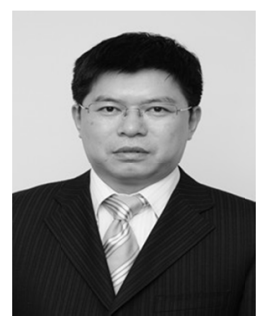

Jianlong Zhao received the Bachelor and $\mathrm{Ph}$. D degrees from Tsinghua University and Shanghai Institute of Microsystem and Information Technology (SIMIT), in 1992 and 1997. Then, he joined State Key Laboratory of Transducer Technology of SIMIT. He is the professor of university of Chinese Academy of Sciences, and chief scientist of national basic research program of China. His research interests include Biomedical Microelectromechanical Systems (BioMEMS) and biosensors.

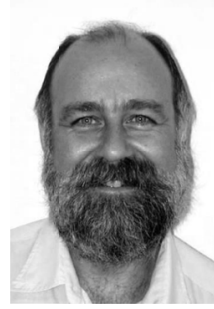

Miguel V. Andrés (M'89) was born in Valencia, Spain, in 1957. He received the Licenciado en Física and Ph.D. degrees from the Universidad de Valencia, Spain, in 1979 and 1985, respectively. From 1983, he served as an Assistant Professor and a Lecturer with the Departamento de Física Aplicada, Universidad de Valencia. He is currently a Professor of Applied Physics with the Departamento de Física Aplicada, Universidad de Valencia, and a member with the Instituto de Ciencia de los Materiales, Universidad de Valencia. Until 1984, he was involved in research on microwave surface waveguides. From 1984 to 1987, he was with the Department of Physics, University of Surrey, U.K., as a Research Fellow, where he was involved in fiber sensors. In 1987, he founded the Optical Fiber Laboratory Group, Universidad de Valencia. His current research interests include waveguide theory (photonic crystal fibers and inhomogeneous waveguides) and optical fiber devices and systems for microwave photonics, all-fiber lasers (active Q-switched and mode-locked fiber lasers) and sensor. 